\title{
Chronic cigarette smoking is linked with structural alterations in brain regions showing acute nicotinic drug-induced functional modulations
}

\author{
Matthew T. Sutherland ${ }^{1 *} \mathbb{D}$, Michael C. Riedel ${ }^{1,2}$, Jessica S. Flannery ${ }^{1}$, Julio A. Yanes ${ }^{3}$, Peter T. Fox ${ }^{4,5,6}$,
} Elliot A. Stein ${ }^{7}$ and Angela R. Laird ${ }^{2}$

\begin{abstract}
Background: Whereas acute nicotine administration alters brain function which may, in turn, contribute to enhanced attention and performance, chronic cigarette smoking is linked with regional brain atrophy and poorer cognition. However, results from structural magnetic resonance imaging (MRI) studies comparing smokers versus nonsmokers have been inconsistent and measures of gray matter possess limited ability to inform functional relations or behavioral implications. The purpose of this study was to address these interpretational challenges through metaanalytic techniques in the service of clarifying the impact of chronic smoking on gray matter integrity and more fully contextualizing such structural alterations.
\end{abstract}

Methods: We first conducted a coordinate-based meta-analysis of structural MRI studies to identify consistent structural alterations associated with chronic smoking. Subsequently, we conducted two additional meta-analytic assessments to enhance insight into potential functional and behavioral relations. Specifically, we performed a multimodal meta-analytic assessment to test the structural-functional hypothesis that smoking-related structural alterations overlapped those same regions showing acute nicotinic drug-induced functional modulations. Finally, we employed database driven tools to identify pairs of structurally impacted regions that were also functionally related via metaanalytic connectivity modeling, and then delineated behavioral phenomena associated with such functional interactions via behavioral decoding.

Results: Across studies, smoking was associated with convergent structural decreases in the left insula, right cerebellum, parahippocampus, multiple prefrontal cortex (PFC) regions, and the thalamus. Indicating a structural-functional relation, we observed that smoking-related gray matter decreases overlapped with the acute functional effects of nicotinic agonist administration in the left insula, ventromedial PFC, and mediodorsal thalamus. Suggesting structuralbehavioral implications, we observed that the left insula's task-based, functional interactions with multiple other structurally impacted regions were linked with pain perception, the right cerebellum's interactions with other regions were associated with overt body movements, interactions between the parahippocampus and thalamus were linked with memory processes, and interactions between medial PFC regions were associated with face processing.

Conclusions: Collectively, these findings emphasize brain regions (e.g., ventromedial PFC, insula, thalamus) critically linked with cigarette smoking, suggest neuroimaging paradigms warranting additional consideration among smokers (e.g., pain processing), and highlight regions in need of further elucidation in addiction (e.g., cerebellum).

\footnotetext{
*Correspondence: masuther@fiu.edu

1 Department of Psychology, Florida International University,

AHC-4, RM 312, 11200 S.W. 8th St, Miami, FL 33199, USA

Full list of author information is available at the end of the article
} 
Keywords: Cigarettes, Nicotine, Addiction, Gray matter, Morphometry, Insula, Mediodorsal thalamus, Ventromedial prefrontal cortex, Cerebellum

\section{Background}

Over the past two decades, neuroimaging has contributed important insight into the structural and functional brain alterations linked with drug abuse in general [1-3] and nicotine addiction in particular [4-6]. For example, such studies have revealed that nicotine administration alters functional brain activity, inducing enhanced activity in some regions involved with attention and cognition (e.g., thalamus, lateral frontoparietal cortices, anterior cingulate cortex $[\mathrm{ACC}]$ ) yet reducing activity in other regions involved with task-irrelevant mental operations (e.g., mind wandering; ventromedial prefrontal cortex, posterior cingulate cortex, parahippocampus) [6-8]. These functional brain alterations may, in part, provide a neurobiological account of the well-documented cognitive enhancing properties of acute nicotine administration [9, 10]. On the other hand, chronic cigarette smokers, compared with nonsmokers, exhibit poorer global cognition and impaired performance on specific measures of working memory, cognitive flexibility, visuospatial learning and memory, and processing speed [11-13]. Aligning with such neurocognitive observations, structural magnetic resonance imaging (MRI) studies have detected reduced gray matter integrity among smokers in multiple discrete brain regions including the prefrontal cortex (PFC), ACC, insula, thalamus, and cerebellum [e.g., 14-17]. Such regional atrophy may result from the deleterious impact of cigarette smoking and/or reflect predisposing neurobiological, neurocognitive, or personality factors.

However, structural MRI results among chronic smokers, to some degree, have been inconsistent. For example, gray matter in the insula of smokers has been reported to be decreased $[15,18,19]$, increased [20], or comparable to that among nonsmokers $[17,21]$. Similarly, whereas some studies have detected smoking-related structural decreases in the ventromedial PFC $[17,19,22]$ or thalamus $[17,21,23]$, others have not $[14,21,24]$. Heterogeneous findings may be the product of cross-sectional designs with modest-to-moderate sample sizes, betweenstudy variability in participant attributes (e.g., varying age ranges, smoking histories, sex ratios, or other sociodemographic characteristics), and/or methodological differences in MRI acquisition or data analysis parameters (e.g., smoothing, registration techniques, or normalization templates). Such issues constrain interpretations from single studies and necessitate the post hoc integration of results from multiple independently conducted studies to better estimate parameters of interest [25].
Accordingly, neuroimaging meta-analytic techniques have been increasingly adopted to delineate spatially convergent results across studies, including identification of consistently observed gray matter alterations among specific phenotypes [26-28].

Although useful for elucidating potential structural alterations among smokers, measures of gray matter are limited in their ability to inform interpretation of functional relations or behavioral implications. Regarding structural-functional relations, a plausible hypothesis is that brain regions showing chronic smoking-related structural alterations overlap those same regions showing acute nicotinic drug-induced functional modulations. One perspective is that the repeated impact of nicotine exposure within discrete brain regions over an individual's extended smoking history culminates in neuroadaptations that may manifest as gray matter perturbations in those same regions. An alternative perspective is that pre-existing structural alterations may render some individuals more susceptible to acute pharmacologic effects and, in turn, to addiction. Regardless of the causative pathway (or combination thereof), integrating structural and functional neuroimaging results may provide insight into the neurobiological processes potentially contributing to the initiation, escalation, and/or maintenance of cigarette smoking. In other words, a multimodal perspective may allow for enhanced interpretation of structural alterations [29, 30]. Regarding structuralbehavioral implications, a frequently posed question not easily answered by considering morphometric outcomes is: what are the behavioral consequences of structural alterations in a particular brain region (or set of regions). As opposed to conjectural discussion of behavioral relevance, empirical approaches to more fully contextualize gray matter alterations are of growing interest [e.g., 31-33]. Such approaches provide an objective means to support behavioral interpretations and/or suggest neuroimaging paradigms warranting additional consideration among a particular phenotype.

In the current study, we aimed to address these interpretational challenges by employing established and emergent meta-analytic techniques to clarify the impact of cigarette smoking on gray matter integrity and to more fully contextualize such morphometic alterations. The specific goals of our study were threefold. First, we sought to identify convergent structural alterations across studies associated with chronic smoking via the well-established, coordinate-based activation likelihood 
estimation (ALE) meta-analytic framework. We operationalized the effects of chronic smoking as gray matter alterations identified in studies utilizing smoker versus nonsmoker comparisons. Second, we tested the structural-functional hypothesis that chronic smoking-related gray matter alterations overlap those same regions showing acute nicotinic drug-induced functional effects via a multimodal meta-analytic assessment. Whereas we operationalized chronic effects as structural alterations identified in smoker versus nonsmoker (i.e., between-subjects) comparisons, we operationalized the acute effects of nicotinic acetylcholine receptor (nAChR) agonist administration as functional alterations indentified in pharmacological neuroimaging studies, the vast majority of which employed within-subjects (i.e., drug versus control condition) comparisons. Third, we sought to provide enhanced structural-behavioral insight via emergent database driven meta-analytic tools, which allow for the characterization of typical patterns of task-based co-activation and associated behavioral phenomenon for user-specified seed regions of interest. Specifically, using smoking-related gray matter alterations to define seed regions, we performed meta-analytic connectivity modeling [33] and behavioral decoding assessments [34, 35] on data archived in an extensive neuroimaging repository (http://www.brainmap.org/) to objectively support behavioral interpretations of structural alterations.

\section{Methods}

\section{Structural MRI study search and selection}

We performed an iterative literature search to compile structural neuroimaging studies interrogating gray matter alterations among chronic cigarette smokers compared with nonsmokers. In the first iteration, we searched the Web of Science (http://www.webofknowledge.com) and PubMed (http://www.pubmed.gov) databases for peer-reviewed articles with the following logical conjunction of terms: ("voxel-based morphometry" OR "morphometry" OR "gray matter density" OR "gray matter volume") AND ("nicotine" OR "tobacco" OR "cigarette" OR "smok"). In a second iteration, we consulted the bibliographies of recent review articles $[5,36]$ and one existing meta-analysis [37] for studies potentially not identified by the database queries. Although a previous meta-analysis has considered the structural impact of chronic smoking, we note that several additional studies have emerged subsequent to that report and highlight our emphasis on structural-functional and structuralbehavioral relations as a further distinguishing characteristic. In a final iteration, we tracked the references of and citations to relevant papers, thereby compiling additional studies.
We included studies in this meta-analysis that: (1) assessed gray matter using structural MRI, (2) reported a set of coordinates (i.e., foci) from a between-subjects contrast comparing smokers to matched nonsmoking participants, (3) reported coordinates in a defined stereotaxic space (i.e., Talairach or Montreal Neurological Institute [MNI]), (4) performed a whole-brain analysis, and (5) provided sufficient information regarding characterization of smoking behaviors (e.g., pack-years, Fagerström Test of Nicotine Dependence [FTND] scores, years smoking, number of cigarettes smoked per day), basic demographics of the study samples (e.g., age, sex, $N$ ), and data analysis strategies (e.g., smoothing parameters, statistical thresholds).

Accordingly, we identified 15 peer-reviewed articles involving 761 cigarette smokers and 1182 nonsmokers (Additional file 1: Figure S1; Table S1) [14, 15, 17-24, 38-42]. Across these 15 identified studies, the smoker samples were on average $41.8 \pm 16.2($ mean $\pm \mathrm{SD})$ years of age and were composed of $40.9 \pm 25.6 \%$ females. At the time of scanning, smokers reported cigarette use for $22.9 \pm 17.0$ years, smoked $17 \pm 4.0$ cigarettes per day, and were moderately nicotine dependent as indicated by FTND scores $(4.5 \pm 1.3$ out of 10$)$. These characteristics were rather consistent across studies and are generally representative of community-based samples of smokers. The nonsmoker samples did not differ from smokers in terms of age (41.1 \pm 17.5 years; $t[14]=-0.9$, $p=0.4)$ or sex $\left(41.6 \pm 25.0 \%\right.$ female: $t[13]^{1}=0.5$, $p=0.6$ ). Most studies mitigated the influence of other drug use by screening via interview and/or urine toxicology on scan days (11 of 15 studies; Additional file 1: Table S2). For each study, we also tabulated information on the type of MRI scanner used and data collection/ analysis parameters (Additional file 1: Table S2). All included studies utilized significance thresholds corrected for multiple comparisons or uncorrected thresholds combined with a spatial extent criterion. These studies distinguished gray matter alterations by the nonsmoker $>$ smoker (i.e., smoking-related decreases) and smoker $>$ nonsmoker directions (i.e., smokingrelated increases). Of those included, 14 studies (78 foci) reported gray matter decreases among smokers and 5 studies ( 10 foci) reported increases. Given the limited number of studies and recent arguments that ALE meta-analyses based on less than 10 experiments/ studies run the risk of obtaining results driven by a single experiment as opposed to identifying convergence across experiments [43], gray matter increases were not considered further.

\footnotetext{
${ }^{1}$ Paired sample $\mathrm{t}$ test. One study did not report the number of females/ males.
} 


\section{Structural impact of chronic cigarette smoking: meta-analytic procedures}

To identify areas of convergent gray matter decreases across studies, we performed a coordinate-based metaanalysis using the revised version [44, 45] of the activation (in this application, Anatomic) likelihood estimation (ALE) algorithm $[26,46]$ as implemented in GingerALE v2.3.4 (http://www.brainmap.org/ale/). ALE is a voxelwise approach for combining neuroimaging results across a collection of experiments/contrasts and thereby identifying locations of statistically significant spatial convergence. The ALE framework models foci as centers of three-dimensional Gaussian probability distributions, thus accounting for spatial uncertainty due to withinand between-study variability. Foci are weighted by study sample size, where larger samples are associated with narrower distributions and smaller samples with wider distributions. We first linearly transformed foci reported in MNI to Talairach space [47] and then generated modeled maps of each individual contrast using their respective foci (paralleling the modeled activation maps of functional MRI [fMRI] meta-analyses). Next, we calculated a voxel-wise ALE score (i.e., the union of all contrasts' modeled maps) quantifying the spatial convergence of structural alterations across the brain. To identify clusters of statistically significant convergence, we compared these obtained ALE scores with those from an empirical null-distribution derived from a permutation procedure [27]. This comparison resulted in nonparametric $p$ value maps, which we then thresholded at a cluster-corrected level ( $p_{\text {corrected }}<0.05$; voxel-level: $p<0.005$, cluster extent: $344 \mathrm{~mm}^{3}$ ) and exported to MANGO (http://www.ric. uthscsa.edu/mango/) for visualization on an anatomical (Talairach) template.

\section{Conjoint chronic smoking-related structural effects and acute drug-induced functional effects: multimodal meta-analytic procedures}

We leveraged previous meta-analytic outcomes regarding the impact of acute $\mathrm{nAChR}$ agonist exposure on brain function to enhance interpretation of structural alterations observed among chronic cigarette smokers. Specifically, in a previous meta-analysis [6] we identified 38 pharmacological fMRI studies that assessed the acute functional effects of nAChR agonist administration (i.e. pharmacologic administration or cigarette smoking) relative to a baseline condition (i.e., placebo administration or smoking abstinence condition) across various cognitive and affective neuroimaging paradigms. The studies meeting selection criteria in that functional meta-analysis involved 796 participants, reported 364 foci from 77 contrasts, and distinguished functional activity modulations by the baseline $>$ drug (i.e., activity decrease) and drug > baseline (i.e., activity increase) directions. We characterized the impact of $\mathrm{nAChR}$ agonists on brain function using the ALE framework (paralleling that described above) and separately identified brain regions showing either convergent activity increases or decreases using a cluster-level corrected threshold $\left(p_{\text {corrected }}<0.05\right)$.

To test the hypothesis that smoking-related gray matter decreases overlap those same regions showing acute drug-induced effects, we conducted a multimodal metaanalytic assessment. Specifically, we performed a conjunction analysis to identify those brain regions, if any, showing statistically significant convergence when considering both: (1) chronic smoking-related structural effects (smokers versus nonsmokers), and (2) acute drug-induced functional effects (nAChR agonist manipulation versus control condition). Employing a conservative minimum statistic conjunction [48], we identified brain regions showing conjoint structural and functional effects by computing the intersection of the two thresholded meta-analytic maps combined with an additional overlap-cluster extent criterion $\left(100 \mathrm{~mm}^{3}\right)$.

\section{Behavioral relevance of structurally impacted regions: meta-analytic connectivity modeling and behavioral decoding}

As gray matter assessments possess limited ability to inform functional or behavioral interpretations, we subsequently employed emergent meta-analytic tools to more fully contextualize the brain circuit-level and behavioral consequences of structural alterations identified among smokers $[28,49,50]$. Specifically, to determine whether structurally impacted brain regions reflect disruption of functionally interrelated neurocircuits, we utilized meta-analytic connectivity modeling (MACM), a validated database driven approach for delineating brain areas that co-activate with a seed region of interest (ROI) across many neuroimaging tasks [33, 51, 52]. This assessment was conducted using the BrainMap database (http://www.brainmap.org/) which is an online repository of over 13,500 neuroimaging contrasts from $\sim 2800$ journal articles (as of January, 2016) archived as threedimensional coordinate-based results $(x, y, z)$ as well as relevant metadata describing the associated experimental design [53-55]. Whereas we utilized the database's archived activation coordinates to characterize the coactivation/connectional profile of structurally-identified ROIs, we utilized the metadata to facilitate behavioral interpretation of smoking-related structural alterations via meta-analytic behavioral decoding. Although the data utilized in these assessments were from healthy participants, they nonetheless offer a useful path to enhance interpretation of observed structural alterations among smokers. These analyses attempt to identify within a 
typical range of function whether pairs of regions interact and, if so, under what behavioral context. If two brain regions are structurally impacted by a certain neuropsychiatric condition and those same regions also appear to interact among healthy participants under a specific behavioral context, one plausible inductive conclusion is that the psychological processes associated with that behavioral context may be disrupted in the neuropsychiatric condition.

Moving beyond isolated regions, we conducted a MACM assessment to characterize the typical pattern of task-based, whole-brain co-activation for each of the structurally-identified ROIs. A MACM assessment aims to identify, across a domain-arching pool of studies interrogating various mental operations and task paradigms, brain areas that simultaneously co-activate with a userspecified seed region. In other words, a MACM assessment identifies brain areas most likely to be activated across all tasks, given activation within a seed. Similar to seed-based resting-state functional connectivity assessments of fMRI data, a MACM identifies those regions that are significantly related to, and presumably interact with the seed. First, we identified experiments in the database that reported one or more activation coordinates within a seed ROI using the Sleuth software application (http://www.brainmap.org/sleuth/). The seeds were $8 \mathrm{~mm}$ radius spheres centered on the voxels with maximum ALE values within each of the smoking-related gray matter loss regions identified above. We conducted separate searches and computed separate MACMs for each of these ROIs. As practiced in previous MACM assessments to achieve sufficient power [e.g., 56], only those ROIs associated with 30 or more experiments in the database were considered for further analyses. We employed $8 \mathrm{~mm}$ radial spheres to equate each ROIs volume and to seek a balance between returning a sufficient number of experiments from the database and minimizing overlap between ROIs that were in close proximity. Next, we extracted the whole-brain coordinates of all foci that co-activated with the seed, constraining this extraction to only activation foci (i.e., no deactivations) reported in studies examining healthy participants (i.e., no intervention or group comparisons). After converting foci reported in MNI to Talairach space [47], we supplied these foci as input to the ALE methodology described above thereby delineating regions of convergent co-activation with the seed when employing a cluster-corrected threshold of $p_{\text {corrected }}<0.05$ (voxel-level: $p<0.001$ ). These thresholded MACM maps for each ROI represent the above-chance probability that identified voxels co-activated with the respective seed across many neuroimaging tasks. To determine whether smoking-related gray matter loss regions reflected disruption of functionally interrelated neurocircuits, we quantified the degree to which one ROI's MACM map intersected with any of the other structurally impacted ROIs. If one ROI's MACM map overlapped at least 50 voxels of any other ROI, those two regions were considered to constitute a circuit-level functional interaction.

In addition to the co-activation coordinates, we also extracted the corresponding BrainMap metadata allowing for the generation of behavioral profiles for each pair of co-activating, and presumably functionally-related, ROIs. The metadata in the database are coded according to a well-defined taxonomy (http://www.brainmap.org/ taxonomy/) cataloguing each contributing study's experimental design, stimulus type, behavioral domain (and subcategory), and paradigm class $[57,58]$. Under this taxonomy, behavioral domains (BD) represent the mental processes interrogated by the primary study's statistical contrasts and comprise the main categories of action, cognition, emotion, interoception, and perception as well as BD subcategories (BD-S; e.g., perception: somesthesispain). Paradigm classes (PC) further categorize the specific task employed (e.g., pain monitoring/discrimination, Go/No-Go). We used these metadata terms to delineate behavioral phenomena linked with the concurrent activation of those pairs of structurally-identified ROIs considered to constitute circuit-level functional interactions in the above MACM assessments.

Specifically, we created behavioral profiles for circuits of interest by performing forward and reverse inference analyses $[34,35]$ on the associated distribution of metadata terms [59, 60]. In the forward inference approach, we tested whether the conditional probability of brain activation given a particular behavioral phenomenon (i.e., BD, $\mathrm{BD}-\mathrm{S}$, or $\mathrm{PC}$ ), $p$ (Activation|Phenomenon), was higher than the baseline probability of brain activation, $p$ (Activation). Baseline activation was defined as the probability of finding a random activation from the database in the region(s) of interest. Significance was established with a binomial test $\left(p_{F D R \text {-corrected }}<0.05\right)$. In the reverse inference approach, we identified the most likely behavioral phenomenon (i.e., BD, BD-S, or PC) given activation in a region. This likelihood, $p$ (Phenomenon|Activation), was derived from $p$ (Activation|Phenomenon) as well as $p$ (Phenomenon) and $p$ (Activation) using Bayes' Rule. Significance was established with a Chi-squared test $\left(p_{\text {FDR-corrected }}<0.05\right)$. Only BD, BD-S, and PC terms that were significant in both the forward and reverse inference approaches are reported. ${ }^{2}$

\footnotetext{
${ }^{2}$ We note that these analyses represent an attempt to relate behavioral phenomena with identified ROIs as opposed to claiming "a unique role" of those ROIs with respect to behavior [34]. In other words, an association of "behavioral phenomenon $x$ to brain region $y$ " obtained from these analyses does not necessarily imply that activity in "region y is limited to behavioral phenomenon $x$ ".
} 


\section{Results}

Structural impact of chronic cigarette smoking

To elucidate structural alterations associated with an extended smoking history, we conducted a meta-analysis identifying consistent gray matter decreases among smokers. This meta-analysis included 78 distinct foci from 14 peer-reviewed studies involving a total of 750 smokers and 1073 nonsmokers (Additional file 1: Table S1). Across these studies, ALE revealed convergent gray matter decreases in 12 distinct clusters, notably in the ventromedial PFC (vmPFC), left insula, and mediodorsal (MD) thalamus, as well as in the medial orbitofrontal cortex (mOFC), ventrolateral PFC (vlPFC), dorsomedial PFC (dmPFC), medial PFC (mPFC), left parahippocampal gyrus, and right cerebellum (Fig. 1; Table 1).

\section{Conjoint chronic smoking-related structural effects and acute drug-induced functional effects}

To delineate regions displaying both structural alterations linked with chronic smoking and functional modulations linked with acute nicotinic agonist administration, we performed a multimodal assessment. Specifically, we conducted a conjunction analysis identifying regions showing both convergent: (1) smoking-related structural decreases (Fig. 1), and (2) nAChR agonist-induced functional decreases or increases [6]. This multimodal assessment identified overlapping structural and functional effects within the vmPFC, left insula, and MD thalamus (Fig. 2; Table 2). Specifically, whereas gray matter decreases in the vmPFC and insula overlapped with clusters of acute drug-induced functional activity decreases (Fig. 2, green; Additional file 1: Figure S2), gray matter decreases in MD thalamus overlapped with drug-induced activity increases (Fig. 2, orange; Additional file 1: Figure S3). We arrived at similar outcomes and the same conclusions when performing this multimodal assessment when considering only functional studies involving nicotine administration (i.e., excluding other nAChR agonists; Additional file 1: Figure S4) and when considering only functional results involving cigarette smokers (Additional file 1: Figure S5).

\section{Behavioral relevance of structurally impacted regions: MACM and behavioral decoding}

To enhance insight into the circuit-level consequences of smoking-related structural alterations, we first performed a MACM assessment thereby identifying clusters

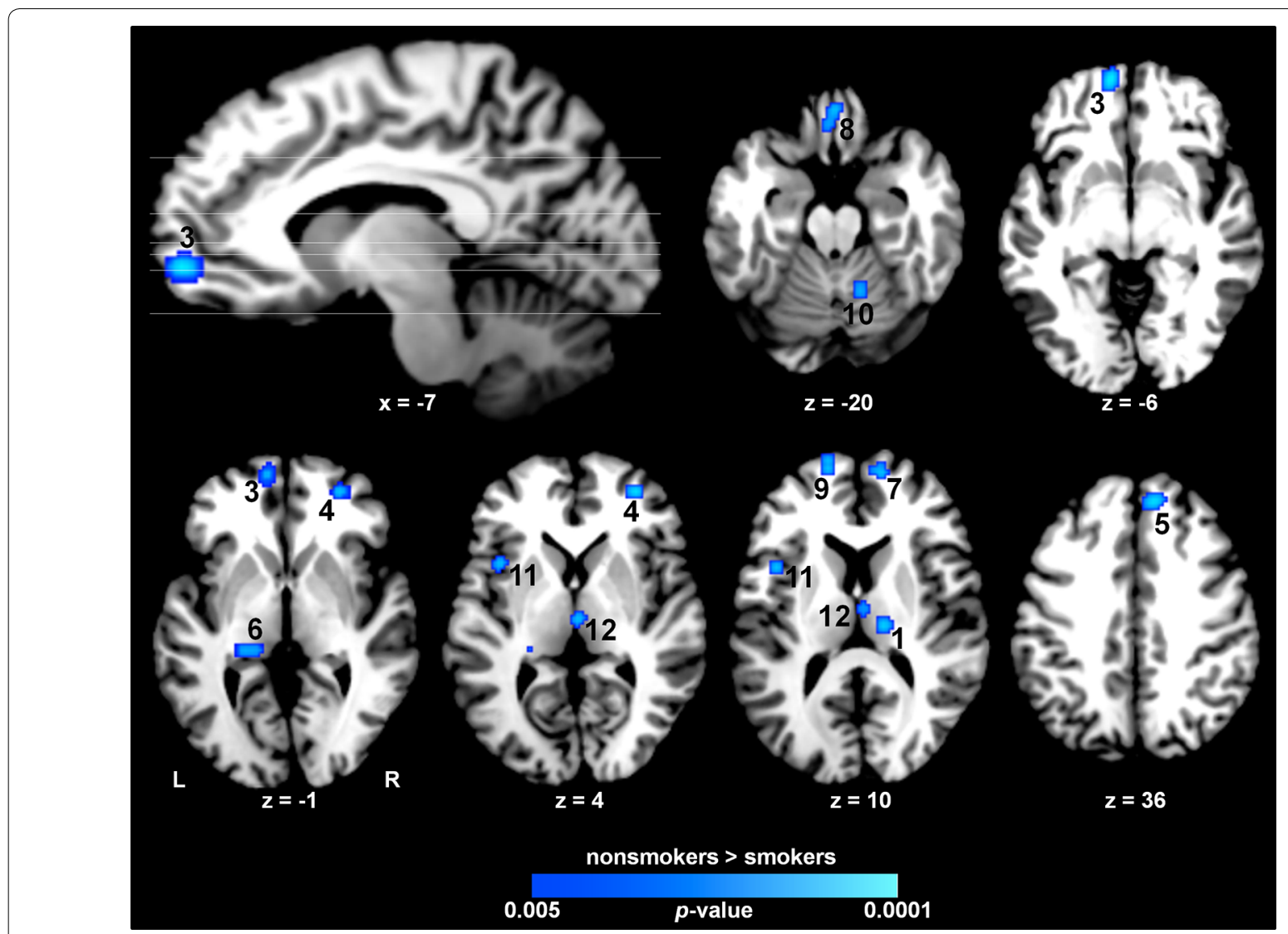

Fig. 1 Structural impact of chronic cigarette smoking. Convergent gray matter decreases among smokers (nonsmokers > smokers) were observed notably in multiple PFC regions, the left insula, thalamus, and cerebellum. Numbering corresponds to coordinates listed in Table 1 
Table 1 Convergent gray matter decreases associated with chronic smoking: cluster coordinates

\begin{tabular}{|c|c|c|c|c|c|c|}
\hline Cluster & Region & & Volume & $\mathrm{X}$ & $\mathrm{Y}$ & Z \\
\hline \multicolumn{7}{|c|}{ Nonsmokers > smokers } \\
\hline 1 & Thalamus (lateral posterior nucleus) & $\mathrm{R}$ & 592 & 16 & -22 & 14 \\
\hline 2 & dmPFC (BA 6) (superior frontal gyrus) & $\mathrm{R}$ & 576 & 16 & 24 & 52 \\
\hline 3 & vmPFC (BA10) (superior frontal gyrus) & L & 568 & -8 & 56 & -4 \\
\hline 4 & VIPFC (BA 10) (middle frontal gyrus) & $\mathrm{R}$ & 480 & 32 & 46 & 4 \\
\hline 5 & dmPFC (BA 8) (medial frontal gyrus) & $\mathrm{R}$ & 480 & 10 & 40 & 36 \\
\hline 6 & Parahippocampal gyrus & L & 432 & -20 & -34 & 0 \\
\hline 7 & mPFC (BA10) (medial frontal gyrus) & $\mathrm{R}$ & 416 & 12 & 58 & 8 \\
\hline 8 & Medial OFC (BA 11) & B & 384 & 2 & 34 & -20 \\
\hline 9 & mPFC (BA10) (medial frontal gyrus) & L & 376 & -14 & 58 & 8 \\
\hline 10 & Cerebellum (dentate) & $\mathrm{R}$ & 368 & 14 & -58 & -20 \\
\hline 11 & Insula (BA 13) & L & 368 & -40 & 8 & 10 \\
\hline 12 & Thalamus (medial dorsal nucleus) & B & 360 & 2 & -18 & 4 \\
\hline
\end{tabular}

Numbering corresponds to brain regions shown in Fig. 1. Coordinates (X, Y, Z) of the clusters' peak voxels are reported in Talairach space. Volume is mm ${ }^{3}$

$B$ bilateral, $R$ right, $L$ left, BA Brodmann area, OFC orbitofrontal cortex, vmPFC ventromedial prefrontal cortex, vIPFC ventrolateral prefrontal cortex, $d m P F C$ dorsomedial prefrontal cortex, MPFC medial prefrontal cortex

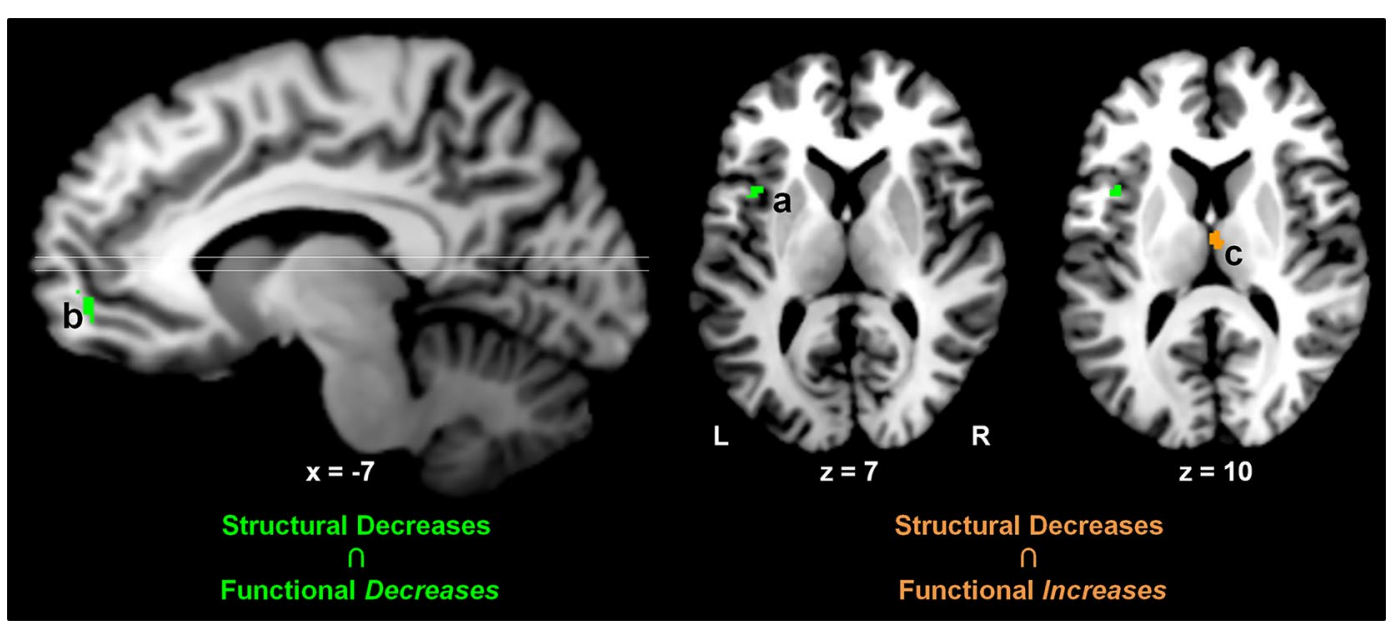

Fig. 2 Conjoint structural and functional effects. Structural alterations (nonsmokers $>$ smokers) overlapped with acute drug-induced activity decreases (baseline > drug) in the insula and ventromedial PFC (green $\mathbf{a}, \mathbf{b}$ ). Structural alterations (nonsmokers > smokers) overlapped with acute drug-induced activity increases (drug > baseline) in the mediodorsal thalamus (orange c). Lettering corresponds to coordinates listed in Table 2. See Additional file 1: Figures S2 and S3 for visualization of overlapping and non-overlapping regions from the structural and functional meta-analyses

Table 2 Conjoint chronic smoking-related structural alterations and acute drug-induced functional activity changes: cluster coordinates

\begin{tabular}{|c|c|c|c|c|c|c|}
\hline Cluster & Region & & Volume & $\mathrm{x}$ & $\mathrm{Y}$ & Z \\
\hline \multicolumn{7}{|c|}{ Gray matter decreases $\cap$ functional decreases } \\
\hline a & Insula (BA 13) & $L$ & 185 & -39 & 7 & 9 \\
\hline$b$ & vmPFC (BA10) (superior frontal gyrus) & $L$ & 103 & -9 & 50 & -3 \\
\hline \multicolumn{7}{|c|}{ Gray matter decreases $\cap$ functional increases } \\
\hline$c$ & Thalamus (medial dorsal nucleus) & B & 142 & 2 & -14 & 11 \\
\hline
\end{tabular}

Lettering corresponds to brain regions shown in Fig. 2. Coordinates $(X, Y, Z)$ of the clusters' peak voxels are reported in Talairach space. Volume is mm ${ }^{3}$ $B$ bilateral, $R$ right, $L$ left, $B A$ Brodmann area, vmPFC ventromedial prefrontal cortex 
of convergent co-activation for each of the structurally impacted ROIs. One region (mOFC) failed to return a sufficient number of experiments from the BrainMap database and was not considered further (Additional file 1: Table S3). The remaining 11 whole-brain MACM maps represent voxels with an above-chance probability of co-activating with the seed when considering various neuroimaging tasks (Fig. 3; Additional file 1: Table S4). For example, the MACM map for: (1) the vmPFC seed (Fig. 3, ROI 3) indentified convergent co-activation within the posterior cingulate cortex, $\mathrm{dmPFC}$, parahippocampus, and inferior frontal gyrus, (2) the left insula seed (Fig. 3, ROI 11) displayed notable co-activation with the right insula, posterior medial prefrontal cortex (encompassing the ACC and supplemental motor area), the thalamus, parietal cortex, and cerebellum, and (3) the $M D$ thalamus seed (Fig. 3, ROI 12) encompassed the posterior medial PFC, bilateral insula, parietal cortex, and cerebellar regions. These and similarly-derived MACM maps delineating networks of task-based co-activation resemble networks identified when considering taskindependent resting-state fMRI data [28, 61-64].

We then further assessed these co-activation maps to determine whether structurally impacted regions reflected functionally interrelated neurocircuits. Those structurally impacted ROIs that also appeared to be functionally related are summarized in Fig. 4a. In this representation, the paths between regions indicate the observation of one ROI intersecting with another ROI's MACM map. For example, the left insula's MACM map (Fig. 4a, ROI 11) overlapped the lateral posterior thalamus (ROI 1), cerebellum (ROI 10), and the MD thalamus (ROI 12) and vice versa (represented by double-headed arrows), whereas the left insula ROI was overlapped by the MACM map derived for the vlPFC seed (ROI 4, single-headed arrow). For the 11 ROIs, 12 unique paths were identified (out of 55; Additional file 1: Figure S6) representing pairs of regions with an above-chance probability of co-activation.

To characterize behavioral phenomena linked with these pairs of functionally-related ROIs, we performed behavioral decoding via forward and reverse inference techniques on the associated BrainMap metadata terms (BD, BD-S, and PC). Those behavioral phenomena significantly associated with co-activation of ROI pairs are represented in Fig. 4b. We highlight four observations from this assessment. First, the left insula's co-activation with multiple other ROIs was linked with aspects of pain processing. Specifically, insula and vlPFC co-activation (Fig. 4b, path: ROI 4-11) was significantly associated with the BD perception (purple), the BD-S somesthesis-pain, and the PC pain monitoring/discrimination. Similarly, concurrent activation of the insula and MD thalamus
(Fig. 4b, path: ROI 11-12) was linked with perception, somesthesis-pain, and pain monitoring/discrimination in addition to the PC recitation/repetition (overt). Insula and lateral posterior thalamus co-activation (Fig. 4b, path: ROI 11-1) was associated with perception and somesthesis-pain. Second, the right cerebellum's co-activation with multiple other ROIs was related to aspects of overt body movements. Specifically, cerebellum and insula coactivation (Fig. 4b, path: ROI 10-11) was associated with the BD action (red), the BD-S execution-speech, and the $\mathrm{PC}$ recitation/repetition. Similarly, concurrent activation of the cerebellum and MD thalamus (Fig. 4b, path: ROI 10-12) was related to action, execution (non-speech), execution-speech, and recitation/repetition in addition to the PCs of flexion/extension and Go/No-Go (i.e., inhibiting an overt body movement). Co-activation of the cerebellum and lateral posterior thalamus (Fig. 4b, path: ROI 10-1) was linked with the BD action, BD-S execution (non-speech), and PC finger tapping/button press. Third, co-activation of the parahippocampus and MD thalamus (Fig. 4b, path: ROI 6-11) was related to the $\mathrm{BD}$ cognition (green), the BD-S memory-explicit, and the PCs cued-explicit and recognition/recall. Lastly, concurrent activation of the vmPFC and mPFC (Fig. 4b, path: ROI 3-9) was associated with the PC face monitoring/ discrimination.

\section{Discussion}

We compiled structural MRI results to clarify the impact of cigarette smoking on gray matter integrity. Our meta-analytic results revealed convergent gray matter decreases among smokers in multiple regions including the prefrontal cortex, insula, thalamus, and cerebellum. Given that such structural measures provide limited insight into functional or behavioral implications, we subsequently performed two additional meta-analytic assessments to more fully contextualize these gray matter decreases. Indicative of a structural-functional relation, we observed via a multimodal assessment that chronic smoking-related structural effects overlapped with the acute functional effects of $\mathrm{nAChR}$ agonist administration in the vmPFC, left insula, and MD thalamus. Suggestive of structural-behavioral implications, we then identified pairs of structurally impacted regions that tended to coactivate across various tasks and delineated behavioral phenomena linked with such co-activation via MACM and behavioral decoding, respectively. These assessments linked the left insula's co-activation with multiple other brain regions to pain perception, the right cerebellum's co-activation with other regions to overt body movement, co-activation of the parahippocampus and MD thalamus with memory processes, and co-activation of medial PFC regions with face processing. 

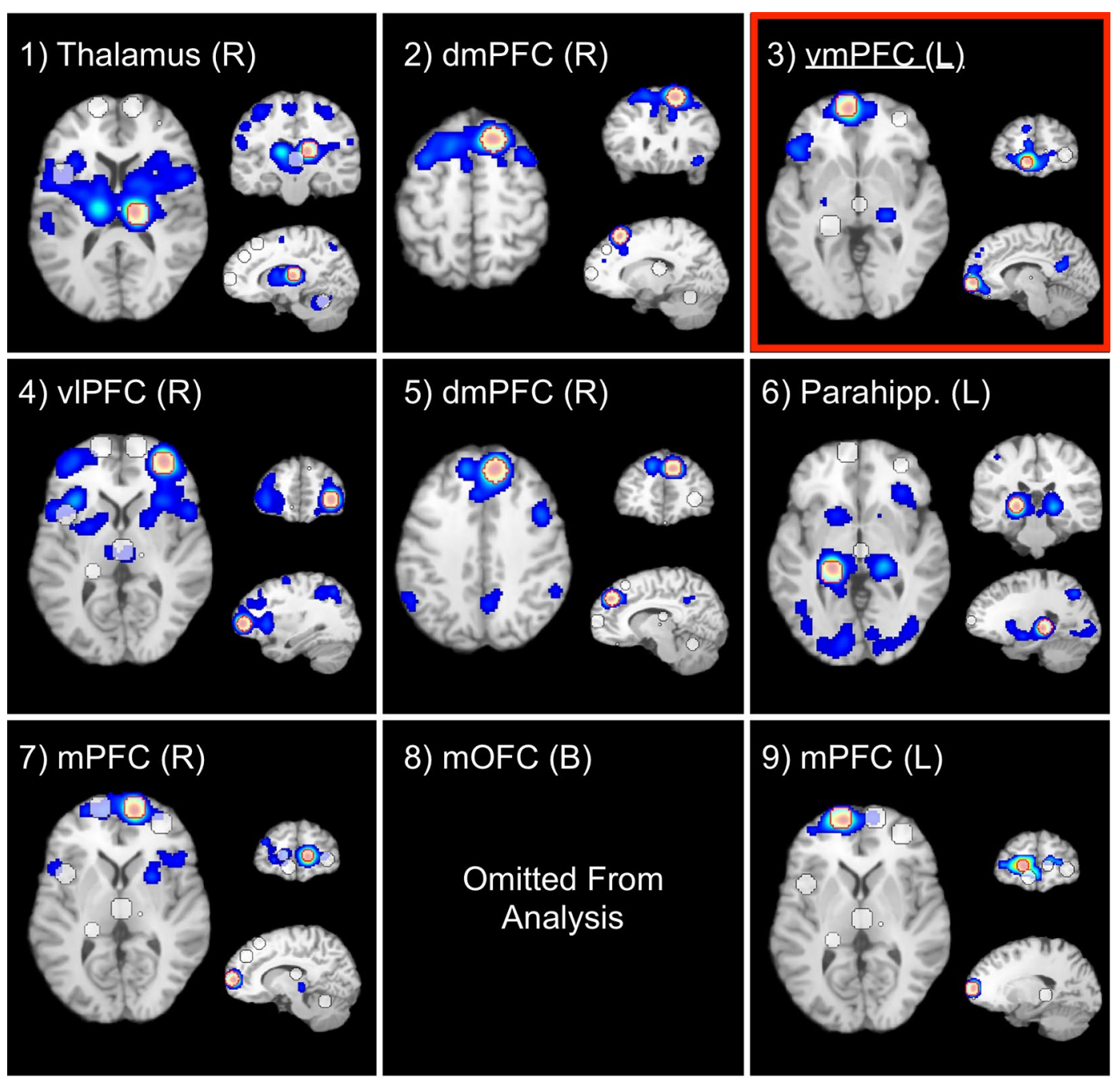

8) $\mathrm{mOFC}(B)$
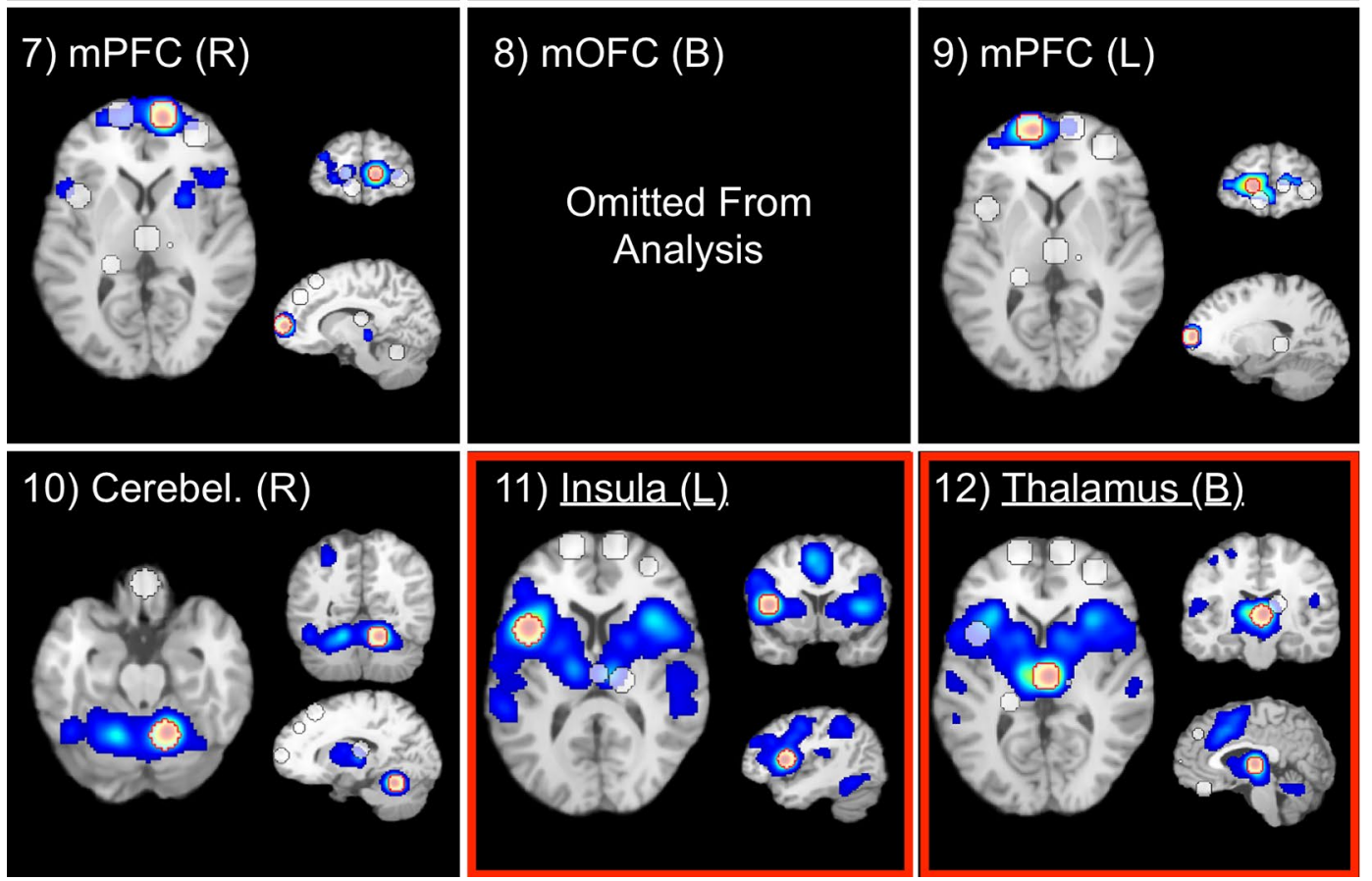

Fig. 3 Meta-analytic connectivity modeling (MACM) maps of task-related co-activation for each structurally impacted ROI. These thresholded MACM maps $\left(p_{\text {corrected }}<0.05\right)$ represent voxels with an above-chance probability of co-activating with the respective seed regions $(8 \mathrm{~mm}$ radius spheres centered on the voxels with maximum ALE values within each smoking-related gray matter loss region). One region (ROI 8; mOFC) failed to return a sufficient number of contrasts from the database and was omitted from further analyses. Numbering corresponds to that in Table 1. The seed ROI of each MACM map is outlined in red. MACM maps for ROls boxed in red are discussed in the main text. See Additional file 1:Table S4 for each seed ROI's co-activation coordinates

Across studies, we observed convergent smokingrelated structural decreases in discrete brain regions notably in the PFC (i.e., ventromedial, ventrolateral, dorsomedial, orbitofrontal), insula, thalamus, and cerebellum. Although we can only speculate on the pathogenesis of such decreases, structural differences between 

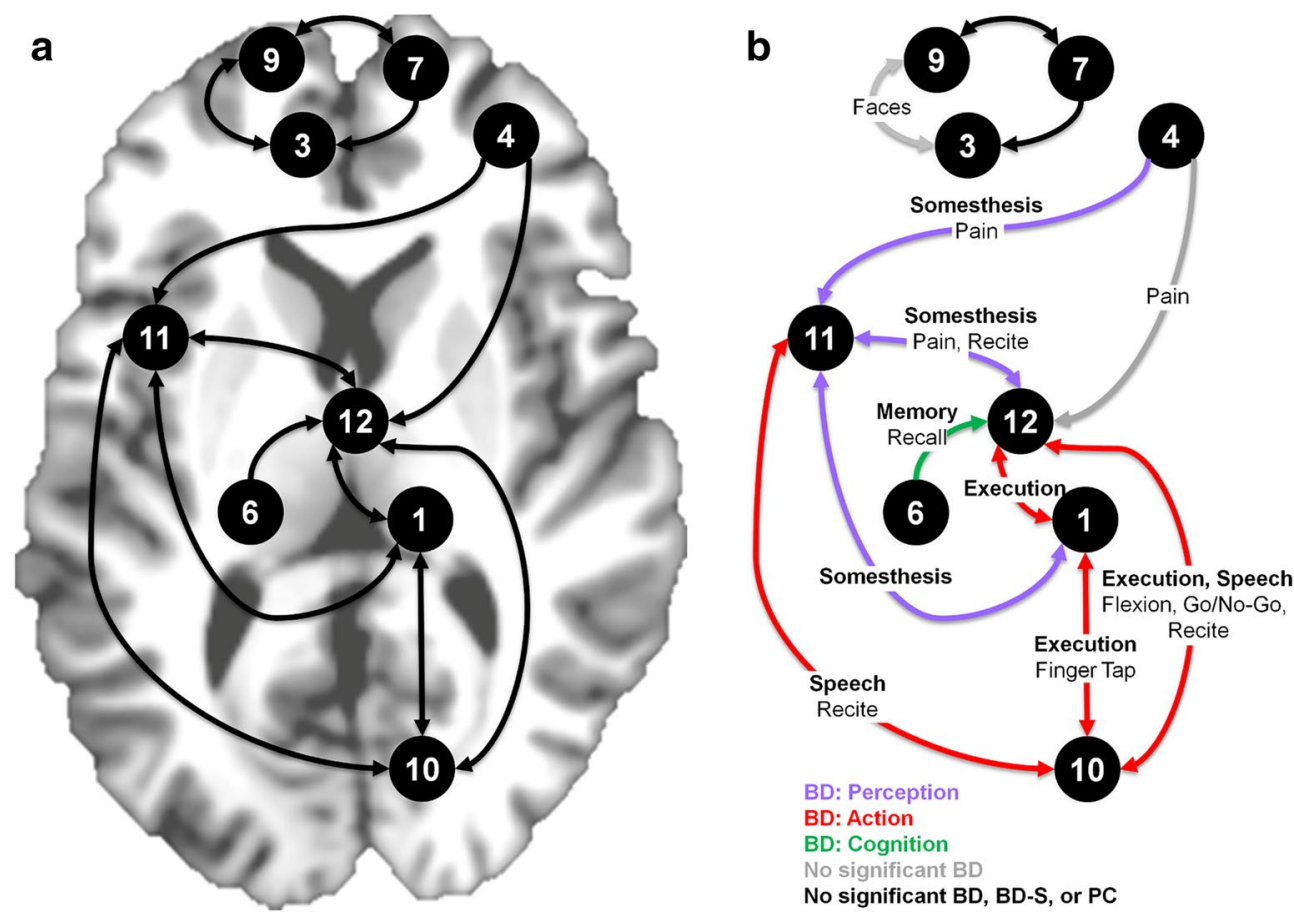

Fig. 4 Pairs of functionally-related ROIs and associated behavioral phenomena. a Functionally-related ROls are connected by paths, the directionality of which indicates that a given ROI (ending point, arrow head) intersected another ROIs MACM map (starting point). We note that if one ROI overlapped another ROI's MACM map, it did not necessarily indicate that the latter ROI overlapped with the former's MACM map. See Additional file 1: Figure $\mathbf{S 6}$ for an alternative representation. b Behavioral phenomena significantly associated with functionally-related ROls were identified by forward and reverse inference assessments of metadata terms. In this representation, significant behavioral domains (BD) are represented by path color (perception [purple], action [red], cognition [green], no significant BD [gray]), significant behavioral domain subcategories (BD-S) are shown along the path in bolded text, and significant paradigm classes $(\mathrm{PC})$ are in standard text. Black paths indicate that no significant associations were detected with any BD, BD-S, or PC

smokers and nonsmokers could be products of predisposing neurobiological risk factors [65], the deleterious impact of cigarette smoking on microvascular functioning [66], neurotoxic events (e.g., oxidative stress, inflammation) associated with many of the $>4000$ compounds present in tobacco smoke [67], and/or be mediated directly or indirectly by nicotine itself. Focusing on the last possibility, nAChRs are ubiquitous throughout the human brain with particularly high densities in the thalamus, insula, and vmPFC $[68,69]$. Widespread upregulation of nAChRs, likely related to receptor desensitization from nicotine exposure, has been observed among smokers [70, 71] and, conversely, smoking cessation associated with decreases in regional nAChR densities [72]. Evidence from preclinical models suggests neurotoxic events and protracted consequences on cholinergic neurotransmission following nicotine exposure particularly during adolescence [73-75]. As such, one plausible mechanistic account is that repeated stimulation of high densities of nAChRs by nicotine may contribute to neurotoxicity within discrete regions and/or increase the vulnerability of those regions to other volatile compounds in tobacco smoke [19] which ultimately manifest as gray matter decreases.

Speaking to such an account, we conducted a multimodal meta-analytic assessment to determine if smoking-related structural decreases overlapped with nicotinic agonist-induced functional modulations and observed conjoint effects in the vmPFC, insula, and MD thalamus. In addition to possessing relatively high nAChR densities, these regions also are critically implicated in addiction-related psychological processes. For example, the vmPFC, in concert with other regions such as the OFC and striatum, is thought to play a role in representing subjective value information, behavioral choice selection, and evaluating outcomes and alterative rewards [76-78]. The insula, a functionally heterogeneous region, is involved with monitoring the physiological state of the body, appears causally related to the initiation, maintenance, and adjustment of attentional control, and likely plays a role in maladaptive decision-making among drug addicts [79-82]. Dysfunction of the MD thalamus, a primary node within a thalamic-PFC-basal ganglia network involved in associative learning, is implicated in the 
transition from goal-directed action (e.g., recreational drug use) to habitual forms of responding (e.g., dysregulated drug seeking) $[83,84]$. Consistent with the notion that structural alterations in these regions are a product of prolonged nicotine/tobacco exposure, group differences between smokers and nonsmokers within, for example, the insula were not detected among an adolescent/young adult participant sample with relatively lower exposure levels $(0.9 \pm 0.7$ pack-years on average) [85] but were observed among similarly aged individuals with relatively higher exposure levels $(4.0 \pm 1.8$ pack-years $)$ [86]. Furthermore, multiple studies have provided evidence for dose-dependent exposure effects on gray matter integrity within the insula $[18,85]$ and vmPFC $[19,22$, $86]$ as well as within the lateral PFC $[14,20]$ and cerebellum [23]. Widespread dose-dependent negative relations between pack-years and cortical thickness were recently corroborated in a large sample of older adults $(N>500)$ with particularly robust effects in the medial and lateral PFC [87]. Interestingly, positive relations between number of years since last cigarette and cortical thickness, suggesting potential recovery with smoking cessation, were also detected in medial PFC and posterior insula regions, amongst others [87]. Our multimodal metaanalytic outcomes add to a growing literature implicating the vmPFC, insula, and thalamus as contributors to the initiation, escalation, maintenance, and/or cessation of cigarette smoking.

Representing one objective approach for providing insight into the behavioral relevance of convergent gray matter decreases among smokers, we utilized emergent meta-analytic tools to delineate structurally impacted ROIs that tended to co-activate and their associated behavioral phenomena. Similar approaches have been used to quantitatively aid interpretation of regions identified via structural assessments or networks commonly observed via resting-state fMRI (i.e., assessments inherently lacking direct behavioral insight) [28,33]. Although we adopted a quantitative approach to infer behavioral relevance of structural alterations, we note that our interpretations remain largely speculative until experimentally verified. We observed that co-activation of the left insula with thalamic and lateral PFC regions were generally linked with pain perception. Dysregulated pain processing is increasingly recognized as a potential barrier to smoking cessation $[88,89]$ and emerging evidence suggests bidirectional relations between pain and smoking behaviors [90, 91]. In laboratory assessments (e.g., cold pressor test), minimally-deprived or abstinent smokers, compared with nonsmokers, exhibit lower pain tolerance and report greater subjective experiences of pain where such pain reports positively correlate with nicotine withdrawal severity [92-94]. As such, nicotine abstinence may contribute to enhanced pain sensitivity that could maintain cigarette smoking via negative reinforcement mechanisms similar to other withdrawal symptoms [95]. Although the insula's role in interoceptive monitoring has been well characterized [80] and higher $\mathrm{nAChR}$ availability in the thalamus, PFC, and cerebellum has been positively correlated with increased pain sensitivity among abstinent smokers [96], relatively little neuroimaging work has focused on the neurobiological processes that may contribute to smoking- or withdrawal-related pain dysregulation. Our meta-analytic outcomes suggest that neuroimaging investigations of pain perception among smokers, with emphasis on the putative functional interactions between the insula and MD thalamus, may be a productive avenue for future research.

We also observed that co-activation of the right cerebellum with insula and thalamic regions were generally linked with overt body movements. Acutely administered, nicotine augments performance in simple motor tasks [9] and increases cerebellar vermis activity during externally paced finger tapping [97]. On the other hand, chronic cigarette smokers perform worse than nonsmokers on neurocognitive measures of fine motor skills and postural stability [11]. Smoking-related decreases in right cerebellum gray matter, identified via an ROI-based approach [16] (i.e., a study not meeting inclusion criteria for the current meta-analysis), further corroborate our observation of convergent decreases in this region. Whereas our outcomes are consistent with the cerebellum's critical role in motor coordination, they also align with contemporary views emphasizing the region's contribution to cognitive processes $[98,99]$ and relevance to drug addiction [100, 101]. For example, we observed that co-activation of the cerebellum and MD thalamus was significantly associated with Go/No-Go paradigms that require inhibitory control. Although current neurobiological models of addiction have yet to provide an integrative account of this region's contribution, altered cerebellar structure and function appears to be a common characteristic across drugs of abuse [100]. Our meta-analytic outcomes serve to further draw attention to the cerebellum in the context of addiction in general and cigarette smoking in particular.

Lastly, co-activation of the parahippocampus and MD thalamus was linked with memory processes and co-activation of medial PFC regions linked with face processing. Regarding the former observation, neurocognitive assessments have consistently identified visuospatial learning and memory deficits among older ( $>60$ years) as well as middle-aged smokers (30-60 years) relative to nonsmokers [11, 12]. Regarding the latter observation, as face processing paradigms often directly or indirectly involve affective components, a plausible interpretation 
is that smoking-related structural effects in medial PFC regions may relate to disrupted emotional processing and/or regulation [102, 103]. Indeed, smokers tend to report elevated negative mood and a reduced ability to self-regulate such states when compared with nonsmokers $[104,105]$.

Our findings should be considered in light of remaining issues. First, the ALE framework is a coordinatebased meta-analytic approach that does not incorporate the size of identified clusters from the primary studies, which leads to less precise representations relative to image-based approaches [106], which, on the other hand, are themselves often less feasible. Second, we only considered gray matter decreases among smokers owing to a small number of reported experiments/foci in the literature with respect to increases. Nonetheless, neuroimaging studies have documented smoking-related volumetric increases in the caudate and putamen $[17,86]$, positive correlations between putamen volume and lifetime nicotine/tobacco exposure [107], and positive correlations between greater striatal volume and more intense cueinduced tobacco craving [108]. Third, we followed previous recommendations that at least 10-15 experiments should be included in an ALE meta-analysis and note that the meta-analysis identifying gray matter decreases among smokers involved 14 studies. Based on recent data simulations [43], closer to $\sim 20$ experiments has been prescribed to achieve sufficient power to detect moderately sized effects. Fourth, meta-analytic results are limited by the methodology commonly employed by the included studies. With respect to the use of T1-weighted MRIs to assess gray matter, we note recent commentaries regarding the potential for drug-induced cerebral blood flow alterations to complicate interpretation of structural outcomes [109, 110]. Given that many studies included in this meta-analysis did not report the time elapsed between last cigarette smoked and MRI data collection (Additional file 1: Table S2), this potential confounding factor cannot be ruled out. Fifth, our behavioral decoding outcomes are limited by the studies archived in the BrainMap database at the time of analysis as well as its associated taxonomy. Sixth, although some studies have begun to characterize the impact of smoking on gray matter in young adult/adolescent smokers $[85,86]$, the concurrent influence of marijuana [111] or alcohol use [112], and sex differences among smokers [17], as more studies accumulate regarding the influence of these and other important factors on gray matter alterations, it will become possible to better characterize the specificity of the meta-analytic effects identified herein. Lastly, it remains for future work, perhaps through multi-site longitudinal investigations, to determine whether regional structural alterations are a cause or consequence of cigarette smoking (or a combination thereof) and the extent to which such alterations recover following cessation.

In sum, cigarette smoking was associated with convergent structural decreases across studies in the PFC, insula, thalamus, and cerebellum. Some of these chronic smoking-related structural effects overlapped with regions showing acute nicotinic drug-induced functional effects. This study highlights the utility of using neuroimaging meta-analytic techniques to compile, synthesize, and inform neuroimaging investigations aiming to elucidate the neurobiological factors underlying the initiation, escalation, maintenance, and/or cessation of cigarette smoking. Collectively, our findings emphasize brain regions (e.g., vmPFC, insula, thalamus) and circuits (e.g., insula-thalamus) linked with chronic smoking, suggest neuroimaging paradigms warranting additional consideration among smokers (e.g., pain processing), and point to regions in need of further elucidation in addiction (e.g., cerebellum).

\section{Additional file}

Additional file 1. Supplementary tables and figures.

\section{Authors' contributions}

MTS and ARL conceived and designed the study. MTS, MCR, JF, JAY contributed to the acquisition, analysis, and visualization of the meta-analytic data. PTF and ARL contributed data analysis tools and technical support. MTS, EAS, $A R L$ contributed to the interpretation of study results. MTS and ARL supervised the study. MTS, PTF, and ARL obtained funding. MTS and ARL supervised the study. MTS drafted the manuscript. All authors critically reviewed the manuscript for important intellectual content. All authors read and approved the final manuscript.

\section{Author details}

${ }^{1}$ Department of Psychology, Florida International University, AHC-4, RM 312, 11200 S.W. 8th St, Miami, FL 33199, USA. ${ }^{2}$ Department of Physics, Florida International University, Miami, FL, USA. ${ }^{3}$ Department of Psychology, Auburn University, Auburn, AL, USA. ${ }^{4}$ Research Imaging Institute, University of Texas Health Science Center, San Antonio, TX, USA. ${ }^{5}$ South Texas Veterans Health Care System, San Antonio, TX, USA. ${ }^{6}$ State Key Laboratory for Brain and Cognitive Sciences, University of Hong Kong, Hong Kong, China. ${ }^{7}$ Neuroimaging Research Branch, National Institute on Drug Abuse, Intramural Research Program, NIH/DHHS, Baltimore, MD, USA.

\section{Acknowledgements}

This work was supported by the National Institute on Drug Abuse (K01-DA037819, MTS) and the National Institute of Mental Health (R01-MH074457 and R56-MH097870, ARL and PTF) of the NIH. EAS is supported by the Intramural Research Program of the National Institute on Drug Abuse. The content is solely the responsibility of the authors and does not necessarily represent the official views of the $\mathrm{NIH}$.

\section{Competing interests}

The authors declare that they have no competing interests.

Received: 23 February 2016 Accepted: 14 May 2016

Published online: 02 June 2016 


\section{References}

1. Koob GF, Volkow ND. Neurocircuitry of addiction. Neuropsychopharm. 2010;35:217-38.

2. Droutman $\mathrm{V}$, Read SJ, Bechara A. Revisiting the role of the insula in addiction. Trends Cogn Sci. 2015;19:414-20.

3. Konova $A B$, Goldstein RZ. Role of the value circuit in addiction and addiction treatment. In: Wilson SJ, editor. The Wiley-Blackwell handbook on the neuroscience of addiction. Hoboken: Wiley-Blackwell; 2015. p. 109-27.

4. Fedota JR, Stein EA. Resting-state functional connectivity and nicotine addiction: prospects for biomarker development. Ann NY Acad Sci. 2015;1349:64-82.

5. Jasinska AJ, Zorick T, Brody AL, Stein EA. Dual role of nicotine in addiction and cognition: a review of neuroimaging studies in humans. Neuropharmacology. 2014;84:111-22.

6. Sutherland MT, Ray KL, Riedel MC, Yanes JA, Stein EA, Laird AR Neurobiological impact of nicotinic acetylcholine receptor agonists: an activation likelihood estimation meta-analysis of pharmacologic neuroimaging studies. Biol Psychiatry. 2015;78:711-20.

7. Menossi HS, Goudriaan AE, de Azevedo-Marques Perico C, Nicastri S, de Andrade AG, D’Elia G, Li CS, Castaldelli-Maia JM. Neural bases of pharmacological treatment of nicotine dependence-insights from functional brain imaging: a systematic review. CNS drugs. 2013;27:921-41.

8. Bentley P, Driver J, Dolan RJ. Cholinergic modulation of cognition: insights from human pharmacological functional neuroimaging. Prog Neurobiol. 2011;94:360-88.

9. Heishman SJ, Kleykamp BA, Singleton EG. Meta-analysis of the acute effects of nicotine and smoking on human performance. Psychopharmacology. 2010;210:453-69.

10. Newhouse PA, Potter AS, Dumas JA, Thiel CM. Functional brain imaging of nicotinic effects on higher cognitive processes. Biochem Pharmacol. 2011;82:943-51.

11. Durazzo TC, Meyerhoff DJ, Nixon SJ. A comprehensive assessment of neurocognition in middle-aged chronic cigarette smokers. Drug Alcohol Depend. 2012;122:105-11.

12. Durazzo TC, Meyerhoff DJ, Nixon SJ. Chronic cigarette smoking: implications for neurocognition and brain neurobiology. Int J Environ Res Public Health. 2010;7:3760-91.

13. Corley J, Gow AJ, Starr JM, Deary IJ. Smoking, childhood IQ, and cognitive function in old age. J Psychosom Res. 2012;73:132-8.

14. Brody AL, Mandelkern MA, Jarvik ME, Lee GS, Smith EC, Huang JC, Bota $\mathrm{RG}$, Bartzokis G, London ED. Differences between smokers and nonsmokers in regional gray matter volumes and densities. Biol Psychiatry. 2004:55:77-84

15. Fritz HC, Wittfeld K, Schmidt CO, Domin M, Grabe HJ, Hegenscheid K, Hosten $\mathrm{N}$, Lotze M. Current smoking and reduced gray matter volume-a voxelbased morphometry study. Neuropsychopharmacology. 2014;39:2594-600.

16. Kuhn S, Romanowski A, Schilling C, Mobascher A, Warbrick T, Winterer G, Gallinat J. Brain grey matter deficits in smokers: focus on the cerebellum. Brain Struct Funct. 2012;217:517-22

17. Franklin TR, Wetherill RR, Jagannathan $K$, Johnson B, Mumma J, Hager $\mathrm{N}$, Rao H, Childress AR. The effects of chronic cigarette smoking on gray matter volume: influence of sex. PLoS ONE. 2014;9:e104102.

18. Stoeckel LE, Chai XJ, Zhang J, Whitfield-Gabrieli S, Evins AE. Lower gray matter density and functional connectivity in the anterior insula in smokers compared with never smokers. Addict Biol. 2015. doi:10.1111/ adb.12262.

19. Hanlon CA, Owens MM, Joseph JE, Zhu X, George MS, Brady KT, Hartwell KJ. Lower subcortical gray matter volume in both younger smokers and established smokers relative to non-smokers. Addict Biol. 2016;21(1):185-95.

20. Zhang X, Salmeron BJ, Ross TJ, Geng X, Yang Y, Stein EA. Factors underlying prefrontal and insula structural alterations in smokers. Neuroimage. 2011;54:42-8.

21. Liao Y, Tang J, Liu T, Chen X, Hao W. Differences between smokers and non-smokers in regional gray matter volumes: a voxel-based morphometry study. Addict Biol. 2012;17:977-80.

22. Kuhn S, Schubert F, Gallinat J. Reduced thickness of medial orbitofrontal cortex in smokers. Biol Psychiatry. 2010;68:1061-5.

23. Gallinat J, Meisenzahl E, Jacobsen LK, Kalus P, Bierbrauer J, Kienast T, Witthaus H, Leopold K, Seifert F, Schubert F, Staedtgen M. Smoking and structural brain deficits: a volumetric MR investigation. Eur J Neurosci. 2006;24:1744-50.

24. Yu R, Zhao L, Lu L. Regional grey and white matter changes in heavy male smokers. PLoS ONE. 2011;6:e27440.

25. Fox PT, Laird AR, Lancaster JL. Coordinate-based voxel-wise meta-analysis: dividends of spatial normalization. Report of a virtual workshop. Hum Brain Mapp. 2005;25:1-5.

26. Laird AR, Fox PM, Price CJ, Glahn DC, Uecker AM, Lancaster JL, Turkeltaub PE, Kochunov P, Fox PT. ALE meta-analysis: controlling the false discovery rate and performing statistical contrasts. Hum Brain Mapp. 2005:25:155-64.

27. Eickhoff SB, Bzdok D, Laird AR, Kurth F, Fox PT. Activation likelihood estimation meta-analysis revisited. Neuroimage. 2012;59:2349-61.

28. Goodkind M, Eickhoff SB, Oathes DJ, Jiang Y, Chang A, Jones-Hagata LB, Ortega BN, Zaiko YV, Roach EL, Korgaonkar MS, et al. Identification of a common neurobiological substrate for mental illness. JAMA Psychiatry. 2015;72:305-15.

29. Radua J, Borgwardt S, Crescini A, Mataix-Cols D, Meyer-Lindenberg A McGuire PK, Fusar-Poli P. Multimodal meta-analysis of structural and functional brain changes in first episode psychosis and the effects of antipsychotic medication. Neurosci Biobehav Rev. 2012;36:2325-33.

30. Cooper D, Barker V, Radua J, Fusar-Poli P, Lawrie SM. Multimodal voxelbased meta-analysis of structural and functional magnetic resonance imaging studies in those at elevated genetic risk of developing schizophrenia. Psychiatry Res. 2014;221:69-77.

31. Nickl-Jockschat T, Kleiman A, Schulz JB, Schneider F, Laird AR, Fox PT, Eickhoff SB, Reetz K. Neuroanatomic changes and their association with cognitive decline in mild cognitive impairment: a meta-analysis. Brain Struct Funct. 2012:217:115-25

32. Nickl-Jockschat T, Schneider F, Pagel AD, Laird AR, Fox PT, Eickhoff SB. Progressive pathology is functionally linked to the domains of language and emotion: meta-analysis of brain structure changes in schizophrenia patients. Eur Arch Psychiatry Clin Neurosci. 2011;261(Suppl 2):S166-71.

33. Laird AR, Eickhoff SB, Li K, Robin DA, Glahn DC, Fox PT. Investigating the functional heterogeneity of the default mode network using coordinate-based meta-analytic modeling. J Neurosci. 2009;29:14496-505.

34. Poldrack RA. Can cognitive processes be inferred from neuroimaging data? Trends Cogn Sci. 2006;10:59-63.

35. Yarkoni T, Poldrack RA, Nichols TE, Van Essen DC, Wager TD. Large-scale automated synthesis of human functional neuroimaging data. Nat Methods. 2011;8:665-70.

36. Wang C, Xu X, Qian W, Shen Z, Zhang M. Altered human brain anatomy in chronic smokers: a review of magnetic resonance imaging studies. Neurol Sci. 2015:36:497-504.

37. Pan P, Shi H, Zhong J, Xiao P, Shen Y, Wu L, Song Y, He G. Chronic smoking and brain gray matter changes: evidence from meta-analysis of voxel-based morphometry studies. Neurol Sci. 2013;34:813-7.

38. Wang K, Yang J, Zhang S, Wei D, Hao X, Tu S, Qiu J. The neural mechanisms underlying the acute effect of cigarette smoking on chronic smokers. PLOS ONE. 2014;9:e102828.

39. Morales AM, Lee B, Hellemann G, O'Neill J, London ED. Gray-matter volume in methamphetamine dependence: cigarette smoking and changes with abstinence from methamphetamine. Drug Alcohol Depend. 2012;125:230-8.

40. Almeida OP, Garrido GJ, Alfonso H, Hulse G, Lautenschlager NT, Hankey GJ, Flicker L. 24-month effect of smoking cessation on cognitive function and brain structure in later life. Neuroimage. 2011;55:1480-9.

41. Almeida OP, Garrido GJ, Lautenschlager NT, Hulse GK, Jamrozik K, Flicker L. Smoking is associated with reduced cortical regional gray matter density in brain regions associated with incipient Alzheimer disease. Am J Geriatr Psychiatry. 2008;16:92-8.

42. Chen X, Wen W, Anstey KJ, Sachdev PS. Effects of cerebrovascular risk factors on gray matter volume in adults aged 60-64 years: a voxelbased morphometric study. Psychiatry Res. 2006;147:105-14.

43. Eickhoff SB, Nichols TE, Laird AR, Hoffstaedter F, Amunts K, Fox PT, Bzdok D, Eickhoff CR. Behavior, sensitivity, and power of activation likelihood estimation characterized by massive empirical simulation. Neuroimage. in press.

44. Eickhoff SB, Laird AR, Grefkes C, Wang LE, Zilles K, Fox PT. Coordinatebased activation likelihood estimation meta-analysis of neuroimaging 
data: a random-effects approach based on empirical estimates of spatial uncertainty. Hum Brain Mapp. 2009;30:2907-26.

45. Turkeltaub PE, Eickhoff SB, Laird AR, Fox M, Wiener M, Fox P. Minimizing within-experiment and within-group effects in Activation Likelihood Estimation meta-analyses. Hum Brain Mapp. 2012;33:1-13.

46. Turkeltaub PE, Eden GF, Jones KM, Zeffiro TA. Meta-analysis of the functional neuroanatomy of single-word reading: method and validation. Neuroimage. 2002;16:765-80.

47. Lancaster JL, Tordesillas-Gutierrez D, Martinez M, Salinas F, Evans A, Zilles K, Mazziotta JC, Fox PT. Bias between MNI and Talairach coordinates analyzed using the ICBM-152 brain template. Hum Brain Mapp. 2007;28:1194-205.

48. Nichols T, Brett M, Andersson J, Wager T, Poline JB. Valid conjunction inference with the minimum statistic. Neuroimage. 2005;25:653-60.

49. Laird AR, Fox PM, Eickhoff SB, Turner JA, Ray KL, McKay DR, Glahn DC, Beckmann CF, Smith SM, Fox PT. Behavioral interpretations of intrinsic connectivity networks. J Cogn Neurosci. 2011;23:4022-37.

50. Reetz K, Dogan I, Rolfs A, Binkofski F, Schulz JB, Laird AR, Fox PT, Eickhoff $\mathrm{SB}$. Investigating function and connectivity of morphometric findingsexemplified on cerebellar atrophy in spinocerebellar ataxia 17 (SCA17). Neuroimage. 2012;62:1354-66.

51. Eickhoff SB, Jbabdi S, Caspers S, Laird AR, Fox PT, Zilles K, Behrens TE. Anatomical and functional connectivity of cytoarchitectonic areas within the human parietal operculum. J Neurosci. 2010;30:6409-21.

52. Robinson JL, Laird AR, Glahn DC, Lovallo WR, Fox PT. Metaanalytic connectivity modeling: delineating the functional connectivity of the human amygdala. Hum Brain Mapp. 2010;31:173-84.

53. Laird AR, Eickhoff SB, Fox PM, Uecker AM, Ray KL, Saenz JJ Jr, McKay DR, Bzdok D, Laird RW, Robinson JL, et al. The BrainMap strategy for standardization, sharing, and meta-analysis of neuroimaging data. BMC Res Notes. 2011;4:349.

54. Laird AR, Eickhoff SB, Kurth F, Fox PM, Uecker AM, Turner JA, Robinson $J \mathrm{~L}$, Lancaster JL, Fox PT. ALE meta-analysis workflows via the BrainMap database: progress towards a probabilistic functional brain atlas. Front Neuroinform. 2009;3:23.

55. Fox PT, Lancaster JL. Opinion: mapping context and content: the BrainMap model. Nat Rev Neurosci. 2002;3:319-21.

56. Riedel MC, Ray KL, Dick AS, Sutherland MT, Hernandez Z, Fox PM, Eickhoff SB, Fox PT, Laird AR. Meta-analytic connectivity and behavioral parcellation of the human cerebellum. Neuroimage. 2015;117:327-42.

57. Fox PT, Laird AR, Fox SP, Fox PM, Uecker AM, Crank M, Koenig SF, Lancaster JL. BrainMap taxonomy of experimental design: description and evaluation. Hum Brain Mapp. 2005;25:185-98.

58. Turner JA, Laird AR. The cognitive paradigm ontology: design and application. Neuroinformatics. 2012;10:57-66.

59. Nickl-Jockschat T, Rottschy C, Thommes J, Schneider F, Laird AR, Fox PT, Eickhoff SB. Neural networks related to dysfunctional face processing in autism spectrum disorder. Brain Struct Funct. 2015;220:2355-71.

60. Cieslik EC, Zilles K, Caspers S, Roski C, Kellermann TS, Jakobs O, Langner R, Laird AR, Fox PT, Eickhoff SB. Is there "one" DLPFC in cognitive action control? Evidence for heterogeneity from co-activation-based parcellation. Cereb Cortex. 2013;23:2677-89.

61. Bzdok D, Heeger A, Langner R, Laird AR, Fox PT, Palomero-Gallagher N, Vogt BA, Zilles K, Eickhoff SB. Subspecialization in the human posterior medial cortex. Neuroimage. 2015;106:55-71.

62. Raichle ME, MacLeod AM, Snyder AZ, Powers WJ, Gusnard DA, Shulman GL. A default mode of brain function. PNAS. 2001;98:676-82.

63. Seeley WW, Menon V, Schatzberg AF, Keller J, Glover GH, Kenna H, Reiss AL, Greicius MD. Dissociable intrinsic connectivity networks for salience processing and executive control. J Neurosci. 2007;27:2349-56.

64. Gu H, Salmeron BJ, Ross TJ, Geng X, Zhan W, Stein EA, Yang Y. Mesocorticolimbic circuits are impaired in chronic cocaine users as demonstrated by resting-state functional connectivity. Neurolmage. 2010;53:593-601.

65. Peters J, Bromberg U, Schneider S, Brassen S, Menz M, Banaschewski T, Conrod PJ, Flor H, Gallinat J, Garavan H, et al. Lower ventral striatal activation during reward anticipation in adolescent smokers. Am J Psychiatry. 2011;168:540-9.

66. Rossi M, Pistelli F, Pesce M, Aquilini F, Franzoni F, Santoro G, Carrozzi L. Impact of long-term exposure to cigarette smoking on skin microvascular function. Microvasc Res. 2014;93:46-51.
67. Swan GE, Lessov-Schlaggar CN. The effects of tobacco smoke and nicotine on cognition and the brain. Neuropsychol Rev. 2007;17:259-73.

68. Picard F, Sadaghiani S, Leroy C, Courvoisier DS, Maroy R, Bottlaender M. High density of nicotinic receptors in the cingulo-insular network. Neuroimage. 2013;79:42-51.

69. Paterson D, Nordberg A. Neuronal nicotinic receptors in the human brain. Prog Neurobiol. 2000;61:75-111.

70. Staley JK, Krishnan-Sarin S, Cosgrove KP, Krantzler E, Frohlich E, Perry E, Dubin JA, Estok K, Brenner E, Baldwin RM, et al. Human tobacco smokers in early abstinence have higher levels of beta2* nicotinic acetylcholine receptors than nonsmokers. J Neurosci. 2006;26:8707-14.

71. Esterlis I, Ranganathan M, Bois F, Pittman B, Picciotto MR, Shearer L, Anticevic A, Carlson J, Niciu MJ, Cosgrove KP, D'Souza DC. In vivo evidence for beta2 nicotinic acetylcholine receptor subunit upregulation in smokers as compared with nonsmokers with schizophrenia. Biol Psychiatry. 2014;76:495-502.

72. Brody AL, Mukhin AG, Stephanie S, Mamoun MS, Kozman M, Phuong J, Neary M, Luu T, Mandelkern MA. Treatment for tobacco dependence: effect on brain nicotinic acetylcholine receptor density. Neuropsychopharmacology. 2013;38:1548-56.

73. Abreu-Villaca Y, Seidler FJ, Tate CA, Slotkin TA. Nicotine is a neurotoxin in the adolescent brain: critical periods, patterns of exposure, regional selectivity, and dose thresholds for macromolecular alterations. Brain Res. 2003;979:114-28.

74. Slotkin TA, Ryde IT, Seidler FJ. Separate or sequential exposure to nicotine prenatally and in adulthood: persistent effects on acetylcholine systems in rat brain regions. Brain Res Bull. 2007;74:91-103.

75. Jain A, Flora SJ. Dose related effects of nicotine on oxidative injury in young, adult and old rats. J Environ Biol. 2012;33:233-8.

76. Bartra O, McGuire JT, Kable JW. The valuation system: a coordinatebased meta-analysis of BOLD fMRI experiments examining neural correlates of subjective value. Neuroimage. 2013;76:412-27.

77. Levy DJ, Glimcher PW. The root of all value: a neural common currency for choice. Curr Opin Neurobiol. 2012;22:1027-38.

78. Hare TA, Camerer CF, Rangel A. Self-control in decision-making involves modulation of the vmPFC valuation system. Science. 2009;324:646-8.

79. Dosenbach NUF, Visscher KM, Palmer ED, Miezin FM, Wenger KK, Kang HSC, Burgund ED, Grimes AL, Schlaggar BL, Petersen SE. A core system for the implementation of task sets. Neuron. 2006;50:799-812.

80. Craig AD. How do you feel-now? The anterior insula and human awareness. Nature Rev Neurosci. 2009;10:59-70.

81. Naqvi NH, Bechara A. The insula and drug addiction: an interoceptive view of pleasure, urges, and decision-making. Brain Struct Funct. 2010;214:435-50.

82. Paulus MP. Decision-making dysfunctions in psychiatry—altered homeostatic processing? Science. 2007;318:602-6.

83. Balleine BW, Morris RW, Leung BK. Thalamocortical integration of instrumental learning and performance and their disintegration in addiction. Brain Res. 2015;1628:104-16.

84. Hogarth L, Balleine BW, Corbit LH, Killcross S. Associative learning mechanisms underpinning the transition from recreational drug use to addiction. Ann NY Acad Sci. 2013;1282:12-24.

85. Morales AM, Ghahremani D, Kohno M, Hellemann GS, London ED. Cigarette exposure, dependence, and craving are related to insula thickness in young adult smokers. Neuropsychopharmacology. 2014;39:1816.

86. Li Y, Yuan K, Cai C, Feng D, Yin J, Bi Y, Shi S, Yu D, Jin C, von Deneen KM, et al. Reduced frontal cortical thickness and increased caudate volume within fronto-striatal circuits in young adult smokers. Drug Alcohol Depend. 2015;151:211-9.

87. Karama S, Ducharme S, Corley J, Chouinard-Decorte F, Starr JM, Wardlaw JM, Bastin ME, Deary IJ. Cigarette smoking and thinning of the brain's cortex. Mol Psychiatry. 2015;20:778-85.

88. Zale EL, Ditre JW, Dorfman ML, Heckman BW, Brandon TH. Smokers in pain report lower confidence and greater difficulty quitting. Nicotine Tob Res. 2014;16:1272-6.

89. Ditre JW, Kosiba JD, Zale EL, Zvolensky MJ, Maisto SA. Chronic pain status, nicotine withdrawal, and expectancies for smoking cessation among lighter smokers. Ann Behav Med. 2016;50:427.

90. Ditre JW, Brandon TH, Zale EL, Meagher MM. Pain, nicotine, and smoking: research findings and mechanistic considerations. Psychol Bull. 2011;137:1065-93. 
91. Parkerson HA, Zvolensky MJ, Asmundson GJ. Understanding the relationship between smoking and pain. Expert Rev Neurother. 2013;13:1407-14.

92. Nakajima M, Al'Absi M. Nicotine withdrawal and stress-induced changes in pain sensitivity: a cross-sectional investigation between abstinent smokers and nonsmokers. Psychophysiology. 2014;51:1015-22.

93. Al'Absi M, Lemieux A, Nakajima M, Hatsukami DK, Allen S. Circulating leptin and pain perception among tobacco-dependent individuals. Biol Psychol. 2015;107:10-5.

94. Pulvers K, Hood A, Limas EF, Thomas MD. Female smokers show lower pain tolerance in a physical distress task. Addict Behav. 2012;37:1167-70

95. Baker TB, Piper ME, McCarthy DE, Majeskie MR, Fiore MC. Addiction motivation reformulated: an affective processing model of negative reinforcement. Psychol Rev. 2004;111:33-51.

96. Cosgrove KP, Esterlis I, McKee S, Bois F, Alagille D, Tamagnan GD, Seibyl JP, Krishnan-Sarin S, Staley JK. Beta2* nicotinic acetylcholine receptors modulate pain sensitivity in acutely abstinent tobacco smokers. Nicotine Tob Res. 2010;12:535-9.

97. Wylie KP, Tanabe J, Martin LF, Wongngamnit N, Tregellas JR. Nicotine increases cerebellar activity during finger tapping. PLOS ONE. 2013;8:e84581.

98. Ramnani N. Frontal lobe and posterior parietal contributions to the cortico-cerebellar system. Cerebellum. 2012;11:366-83.

99. Stoodley CJ. The cerebellum and cognition: evidence from functional imaging studies. Cerebellum. 2012;11:352-65.

100. Moulton EA, Elman I, Becerra LR, Goldstein RZ, Borsook D. The cerebellum and addiction: insights gained from neuroimaging research. Addict Biol. 2014;19:317-31.

101. Miquel M, Vazquez-Sanroman D, Carbo-Gas M, Gil-Miravet I, SanchisSegura C, Carulli D, Manzo J, Coria-Avila GA. Have we been ignoring the elephant in the room? Seven arguments for considering the cerebellum as part of addiction circuitry. Neurosci Biobehav Rev. 2016;60:1-11.

102. Lindquist KA, Wager TD, Kober H, Bliss-Moreau E, Barrett LF. The brain basis of emotion: a meta-analytic review. Behav Brain Sci. 2012:35:121-43.
103. Foland-Ross LC, Altshuler LL, Bookheimer SY, Lieberman MD, Townsend J, Penfold C, Moody T, Ahlf K, Shen JK, Madsen SK, et al. Amygdala reactivity in healthy adults is correlated with prefrontal cortical thickness. J Neurosci. 2010;30:16673-8.

104. Lyvers M, Carlopio C, Vicole Bothma H, Edwards MS. Mood, mood regulation, and frontal systems functioning in current smokers, longterm abstinent ex-smokers, and never-smokers. J Psychoact Drugs. 2014;46:133-9.

105. Lyvers M, Carlopio C, Bothma V, Edwards MS. Mood, mood regulation expectancies and frontal systems functioning in current smokers versus never-smokers in China and Australia. Addict Behav. 2013;38:2741-50.

106. Salimi-Khorshidi G, Smith SM, Keltner JR, Wager TD, Nichols TE. Metaanalysis of neuroimaging data: a comparison of image-based and coordinate-based pooling of studies. Neuroimage. 2009;45:810-23.

107. Das D, Cherbuin N, Anstey KJ, Sachdev PS, Easteal S. Lifetime cigarette smoking is associated with striatal volume measures. Addict Biol. 2012;17:817-25.

108. Janes AC, Park MT, Farmer S, Chakravarty MM. Striatal morphology is associated with tobacco cigarette craving. Neuropsychopharmacology. 2015:40:406-11.

109. Franklin TR, Wang Z, Shin J, Jagannathan K, Suh JJ, Detre JA, O'Brien CP, Childress AR. A VBM study demonstrating 'apparent' effects of a single dose of medication on T1-weighted MRIs. Brain Struct Funct. 2013;218:97-104

110. Franklin TR, Wetherill RR, Jagannathan $K$, Hager N, O'Brien CP, Childress AR. Limitations of the use of the MP-RAGE to identify neural changes in the brain: recent cigarette smoking alters gray matter indices in the striatum. Front Hum Neurosci. 1052;2014:8.

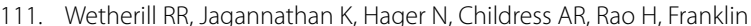
TR. Cannabis, cigarettes, and their co-occurring use: disentangling differences in gray matter volume. Int J Neuropsychopharmacol. 2015;18:pyv061

112. Luhar RB, Sawyer KS, Gravitz Z, Ruiz SM, Oscar-Berman M. Brain volumes and neuropsychological performance are related to current smoking and alcoholism history. Neuropsychiatric Dis Treat. 2013;9:1767-84.

\section{Submit your next manuscript to BioMed Central and we will help you at every step:}

- We accept pre-submission inquiries

- Our selector tool helps you to find the most relevant journal

- We provide round the clock customer support

- Convenient online submission

- Thorough peer review

- Inclusion in PubMed and all major indexing services

- Maximum visibility for your research

Submit your manuscript at www.biomedcentral.com/submit 